\title{
Drug Repositioning: A Faster Path to Drug Discovery
}

\author{
Vishwani Persaud-Sharma and Shu-Feng Zhou*
}

Department of Pharmaceutical Sciences, College of Pharmacy, University of South Florida, Tampa, FL 33612, USA

\begin{abstract}
With the consistent decline in drug production over the past years, pharmaceutical companies strive to find alternative means to manufacture and market therapeutic drugs at lightening speeds. In an attempt to negate high manufacturing costs and lengthy timelines, researchers and clinicians look to repositioning as a means of recreating new drugs from older ones with novel functionalities and better efficacy. Successfully repositioned drugs such as Viagra have hit the market with exponential pharmaceutical revenue records made at a fraction of the cost versus de novo methodologies paving the way for drug prospects that follow in similar footsteps.
\end{abstract}

\section{What is Drug Repositioning and why is it Beneficial?}

With the considerable variation in acquired ailments and new founded resilience and mutations observed in bacterial and viral strands of toxins world-wide, pharmaceutical companies strive to derive and elucidate simplistic and cost effective methods for the actualization of drugs that promote efficacy, efficiency, and targeted drug delivery. On a yearly basis, biopharmaceutical companies face numerous gaps in productivity in terms of delayed output that lags far behind the prodigious rise in pharmaceutical spending geared towards research and development [1]. Researchers have estimated that the cost of developing a single new drug from scratch may induce a deficit greater than US $\$ 800$ million with the projected duration of development to be over the course of ten to seventeen years [2]. According to recent studies [3], there are two main justifications attributed to the decrease in safe and marketable drugs that tend to linger in the subsequent processes that ensues the journey to retail shelves. Primarily, conservative drug development strategies function to invent novel therapeutic targets coupled with substances that alter target activity which is typically prolonged, expensive, and involves experimental risk and lengthy clinical cross-referencing [3]. Secondarily, Dudley et al. [3] suggests that productivity reduction is noted by the absence of systemic evaluation in addition to indications at the target site as well as throughout the phase of development which affects the drugs marketability. In an attempt to minimize superfluous time and money spent in an effort to manufacture, establish, and market new drugs that may only exhibit minor alterations with reference to composition or esthetic features, scientists have established a modification system that has long served to inculcate faster, more reliable methods to enhance drug manufacturing termed drug repositioning or drug repurposing.

The premise of drug repositioning functions in terms of the methodologies implemented in the quest of discovering new uses for existing drugs [4]. Drug coincidence or 'serendipity' as termed by some authors, arise from unintentional mishaps in the drug remodeling process which can be exemplified in iconic cases of drugs such Sildenafil, commonly known as Viagra and Thalidomide commonly known as Thalomid [5]. While complete de novo synthesis of these drugs would have endured an exorbitant financial pitfall, drug remodeling enabled synthesis of these drugs for a fraction of the cost with revenues surmounting that of costly technological approaches. Statistical compilation recapitulated by Dudley et al. [3] indicate that gross revenue attributed to Thalidomide, a drug repositioned for multiple myeloma, exceeded US\$271 million in 2003 whereas the drug repositioned for erectile dysfunction Sildenafil or Viagra exhibited US $\$ 1.88$ billion within the same year.
On a general platform, a slew of benefits account for the widely accepted use of drug repositioning. Apart from the staggering manufacturing cost and time reduction, drug repositioning facilitates novel drug applications that amplify patent life, thereby projecting investment return throughout the development process coupled with a much higher rate of success [6]. Drug shelving by pharmaceutical companies can be eliminated where approximately two hundred substances can now be solicited for alternative indications despite failure in previous clinical trials with better financing available for smaller companies. The benefits of drug repositioning for patients are evident in that newly arising ailments can be treated that was otherwise deemed a highly experimental pathway in addition to the use of established side effects, pharmacokinetics, and drug interactions standardized by previous studies where specific repositioning value are exhibited in the associated references [6].

Support for the benefits of drug repositioning has not only taken hold of the pharmaceutical market but is also becoming established in many academic settings in terms of translational medicine and nonprofit research organizations; however, the National Institute of Health (NIH) has yet to launch official consent in the form of repositioning specific funded grants and allowances [7]. Privately funded research awards have been issued and can be exemplified by the Michael J. Fox Foundation that issued the dispersal of a total of \$2.4 million for undertaking a total of six repositioning projects predominantly geared towards research for Parkinson's disease among other various causes [7].

\section{How Drug Repositioning Works}

On a bioinformatics platform, drug repositioning can be classified as (i) drug-derived, which utilizes repositioning mechanisms from

${ }^{*}$ Corresponding author: Shu-Feng Zhou, MD, PhD, Professor, Associate Vice President for Global Medical Development, Associate Dean for International Research, \& Chair of the Department of Pharmaceutical Sciences, College of Pharmacy, University of South Florida, 12901 Bruce B. Downs Boulevard, Tampa, Florida 33612, USA, Tel: 813 974-6276; Fax: 813 905-9885; E-mail: szhou@health.usf.edu

Received October 25, 2012; Accepted October 26, 2012; Published October 27, 2012

Citation: Persaud-Sharma V, Zhou SF (2012) Drug Repositioning: A Faster Path to Drug Discovery. Adv Pharmacoepidem Drug Safety 1:e117. doi:10.4172/2167 1052.1000e117

Copyright: (C) 2012 Persaud-Sharma V, et al. This is an open-access article distributed under the terms of the Creative Commons Attribution License, which permits unrestricted use, distribution, and reproduction in any medium, provided the original author and source are credited. 
chemical or pharmaceutical directives, or (ii) disease-derived, which instills directives from a disease management, pathology, or symptomatology perspective [3]. Primarily, drug-derived pharmaceuticals aim to target potential marketable drugs that are deemed 'safe' in Phase I clinical trials but have been dejected based on efficacy roadblocks in subsequent Phases II and III of the clinical trials [8]. Secondarily, disease-derived repositioning targets chronic disease studies that do not have effectively safe therapeutic regimes with particular attention directed towards long-term treatment of these diseases and disease stabilization [8]. Bioinformatic analysis aids in the discovery of these novel drug interactions where in silico drug repositioning is facilitated by the NCGC Pharmaceutical Collection (NPC) that encompasses approved small molecule drugs that can be observed and analyzed via ultra high through-put screening assays to schematically decipher and select or deselect repositioning prospects relevant for human diseases [5]. Drug-derived repositioning investigations can be regulated and isolated by various elements used for refining experimental investigations such as molecular isolations by (i) chemical similarity known as the quantitative relationships between chemical structures and biochemical activity (QSAR), (ii) similarities in molecular drug activity can be where active compounds undergo perturbation through the compounds' mechanism of action (MOA) as a result of biological system exposure that can be modified and assessed through the construction of Connectivity Maps, and finally (iii) molecular docking used to predict characteristic interactions between the existing compounds and novel molecular targets by means of target-by-target assessment or ligand-target pathways for prospective repositioning strategies [3]. On the other hand, diseasederived investigation of drug repositioning encompasses (i) associative indication transfer which uses a 'guilt by association' approach in an attempt to elucidate novel drug interactions via similarity in the number of therapies the drug shares with another established drug for a given disease, (ii) shared molecular pathology where a drug can be repositioned from the indication of a different drug in the advent that both substances exhibit parallel molecular pathophysiology with response to therapeutic effect, and (iii) similarities in side effects in which the drug is repurposed via the side effects characterizing unintentional consequences of the actions if the drug which hint towards biological activity of the drug [3].

\section{A Glimpse into Current Repositioning Studies and Future Directives}

In today's day and age, drug repositioning is quickly making its way to mainstream use world- wide. Technologically, pharmaceutical companies have utilized such mechanisms to explore and implement the quick turn over of drug manufacturing via the novel technologies associated with drug repositioning. As time progresses, a rapid increase in scientific and clinical manufacturing methodologies seem to emerge with equal attention geared towards the development of both drugderived repositioning as well as disease-derived repositioning. As exemplified by Iorio et al. (2010) Haupt and Schroeder Lee et al. Harrison, and Yang and Agarwal [6,9-12] to pinpoint a few researchers among the inclusive past three years who have made recent contributions to the drug-derived repositioning studies, substantial pharmaceutical and molecular studies have been established with direct focus towards the investigation of newly inculcated applications of drug-derived repositioning strategies with predominant focus on further MOA observations. Methodologies commonly associated with drug MOA investigation typically include chemical structure analysis, transcriptional responses post treatment, as well as text mining [9]. Despite the usefulness of these readily available techniques, due to insufficiency in data accumulation the author developed an automated method of investigation to isolate similarities and discordances in gene expression profiles post treatment exposure amidst differing parameters with specifics attributed to the generation of a pharmacological drug network that consisted of 1,302 drugs or nodes as well as 41,047 edges denoting drug concordances targeting substances with parallel MOAs or functioning on similar pathways in hopes of tracking previously undisclosed pathways [9]. On the same note, studies conducted by Yang and Agarwal [12] examine the in silio functionalities to determine a given drug's off-target effect exhibited by gene expression perturbation as well as off-phenotype effects such as detrimental/adverse drug reactions demonstrated by undesired clinical side-effects. The outcome of the study included the formulation of a disease based clinical side-effects database created from extractions of the drug side effects database through the use of SPIDER labeled drugs coupled with drug-disease relations obtained from PharmCKG in conjunction with drug repositioning methods that were established in terms of the Side-Effectome (DRoSEf) database [12]. In an attempt to assist researchers explore the variable outcomes of in silico drug repositioning prior to validation steps, Liu et al. [8] developed a comparative Drugs of New Indication (DNI) database to aid in the exploration and establishment of undocumented drug indications. The experimental investigations of a database termed PharmDB which utilized an established binary links between drugs, disease, and proteins with the assistance of established drug-disease relationships [10]. Finally, Harrison [11] reviewed two methods of analysis used to examine gene expression data in terms of microarray data in terms of 100 diseases characterized by from the Gene Expression Omnibus as well as data obtained from human cancer cell lines with treatment associated with 164 small molecules which lead to the utilization of significance analysis of microarrays specific to molecular signature determination.

Studies geared towards the investigation of disease-derived drug repositioning methodologies facilitate those drug instigations specific for a given disease or ailment. As depicted by Jin et al. [13], researchers strive to investigate the potential therapeutics for off-target drugs that can be repositioned for use in surrogate treatment for cancer with specific aim in creating a defined cancer-signaling bridge (CSB) network targeted towards breast cancer, prostate cancer cells, and promyelocytic leukemia cells. Studies conducted by Kinnings et al. [14] elucidated novel methodologies leading to the magnification of cross-reactivity between target drug families in an attempt to reposition drugs used for Parkinson's disease to aid in the treatment of multi-drug resistant (MDR) and extensively drug resistant (EDR) tuberculosis. Similarly, literature in support of the prospect for drug repositioning of MDR tuberculosis was presented by Palomino and Martin [15]. On a broader spectrum, the report presented by Iorio et al. (2012) [16] depicted gene expression signatures which revealed uncharacteristic drug-disease and drug-drug interactions that paralleled alternative signatures and the relevant studies that surround microarray analysis leading to drug repositioning prospects. Studies conducted by Sanseau et al. [17] substantiated the claim that genome-wide association studies (GWAS) investigations are useful in developing new methodologies that aid in the prediction of the biology of diseases functioning in the translational exploration of drug discovery and repositioning leading to the impact of 63 matches of the genetics of drug discovery from information obtained from clinical trials. Finally, literary compilation derived by Lussier and Chen [18] have elucidated other data bases similar to GWAS such as 
Citation: Persaud-Sharma V, Zhou SF (2012) Drug Repositioning: A Faster Path to Drug Discovery. Adv Pharmacoepidem Drug Safety 1:e117. doi:10.4172/2167-1052.1000e117

Page 3 of 3

gene set enrichment analyses (GSEAs) among others which observe a non-biased analysis of gene expression with observations of the entire genome.

While the emergence of drug reposition is currently taking center stage among the vast, time consuming, and monetarily exorbitant methodologies of de novo drug synthesis used among pharmaceutical companies today, much thought has been established in the actualization of repositioning as a permanent solution for enhanced drug indications of existing therapeutics. With the newly established platform resonating successfully repositioned drugs such as Viagra and Thalomid, researchers and clinicians are intrigued by the prospect of creating new drugs from older ones. Keeping the cliché that all that glitters in not gold in mind, researchers continue to make steady progress in the new development of drugs that can save the lives of millions by simple methodologies of drug repositioning. Through the use of bioinformatics, drug-derived, and disease-derived repositioning methodologies, pharmaceuticals have seen a vast increase in sales and amplified manufacturing times with therapeutics geared towards targeted treatment of orphaned and well established illness. So what is the future of drug repositioning? You decide, the possibilities are endless and it can save lives!

\section{References}

1. Ashburn TT, Thor KB (2004) Drug repositioning: identifying and developing new uses for existing drugs. Nat Rev Drug Discov 3: 673-683.

2. Pan SY, Pan S, Yu ZL, Ma DL, Chen SB, et al. (2010) New perspectives on innovative drug discovery: an overview. J Pharm Pharm Sci 13: 450-471.

3. Dudley JT, Deshpande T, Butte AJ (2011) Exploiting drug-disease relationships for computational drug repositioning. Brief Bioinform 12: 303-311.

4. Tobinick EL (2009) The value of drug repositioning in the current pharmaceutical market. Drug News Perspect 22: 119-125.

5. Bisgin H, Liu Z, Kelly R, Fang H, Xu X, et al. (2012) Investigating drug repositioning opportunities in FDA drug labels through topic modeling. BMC Bioinformatics Suppl 15: S6.

6. Haupt VJ, Schroeder M (2011) Old friends in new guise: repositioning of known drugs with structural bioinformatics. Brief Bioinform 12: 312-326.

7. Dolgin $E$ (2011) Nonprofit disease groups earmark grants for drug repositioning Nat Med 17: 1027.

8. Liu Z, Fang H, Reagan $\mathrm{K}, \mathrm{Xu} \mathrm{X}$, Mendrick DL, et al. (2012) In silico drug repositioning - what we need to know. Drug Discov Today S13596446(12)00299-1.

9. Iorio F, Bosotti R, Scacheri E, Belcastro V, Mithbaokar P, et al. (2010) Discovery of drug mode of action and drug repositioning from transcriptional responses. Proc Natl Acad Sci U S A 107: 14621-14626.

10. Lee HS, Bae T, Lee JH, Kim DG, Oh YS, et al. (2012) Rational drug repositioning guided by an integrated pharmacological network of protein, disease and drug. BMC Syst Biol 6: 80

11. Harrison C (2011) Signatures for drug repositioning. Nat Rev Genet 12: 668

12. Yang L, Agarwal $P$ (2011) Systematic drug repositioning based on clinical sideeffects. PLoS One 6: e28025.

13. Jin G, Fu C, Zhao H, Cui K, Chang J, et al. (2012) A novel method of transcriptional response analysis to facilitate drug repositioning for cancer therapy. Cancer Res 72: 33-44

14. Kinnings SL, Liu N, Buchmeier N, Tonge PJ, Xie L, et al. (2009) Drug Discovery Using Chemical Systems Biology: Repositioning the Safe Medicine Comtan to Treat Multi-Drug and Extensively Drug Resistant Tuberculosis. PLoS Comput Biol 5: e1000423.

15. Palomino JC, Martin A (2012) Is repositioning of drugs a viable alternative in the treatment of tuberculosis? J Antimicrob Chemother.

16. Iorio F, Rittman T, Ge H, Menden M, Saez-Rodriguez J (2012) Transcriptional data: a new gateway to drug repositioning? Drug Discov Today.

17. Sanseau P, Agarwal P, Barnes MR, Pastinen T, Richards JB, et al. (2012) Use of genome-wide association studies for drug repositioning. Nat Biotechnol 30 : 317-320.

18. Lussier YA, Chen JL (2011) The emergence of genome-based drug repositioning. Sci Transl Med 3: $96 \mathrm{ps} 35$. 